$$
\begin{aligned}
& \text { Pontifícia Universidade C Cólólca } \\
& \text { DO RIO DE JANEIRO }
\end{aligned}
$$

Marcus Vinicius Pereira de Souza

\title{
Uma Abordagem Bayesiana para o Cálculo dos Custos Operacionais Eficientes das Distribuidoras de Energia Elétrica
}

\section{Tese de Doutorado}

Tese apresentada ao Programa de Pós-graduação em Engenharia Elétrica do Departamento de Engenharia Elétrica da PUC-Rio como parte dos requisitos parciais para obtenção do título de Doutor em Engenharia Elétrica.

Orientador: Prof. Reinaldo Castro Souza

Rio de Janeiro

Maio de 2008 


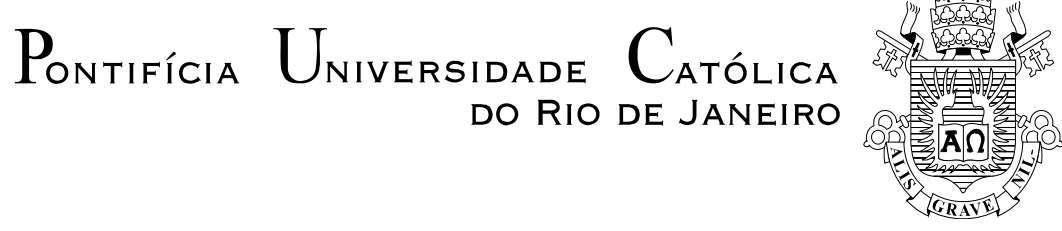

Marcus Vinicius Pereira de Souza

\begin{abstract}
Uma Abordagem Bayesiana para o Cálculo dos Custos Operacionais Eficientes das Distribuidoras de Energia Elétrica
\end{abstract}

Tese apresentada como requisito parcial para obtenção do grau de Doutor pelo Programa de Pós-Graduação em Engenharia Elétrica do Departamento de Engenharia Elétrica do Centro Técnico Científico da PUC-Rio. Aprovada pela Comissão Examinadora abaixo assinada.

Prof. Reinaldo Castro Souza

Orientador

Departamento de Engenharia Elétrica - PUC-Rio

Prof. Madiagne Diallo

Co-orientador

Departamento de Engenharia Industrial

Prof. José Francisco Moreira Pessanha

CEPEL

Prof. Tufi Machado Soares

Universidade Federal de Juiz de Fora

Profa. Mariane Branco Alves

UERJ

Prof. Marcos Azevedo da Silveira Departamento de Engenharia Elétrica - PUC-Rio

Prof. José Eugenio Leal Coordenador Setorial do Centro

Técnico Científico

Rio de Janeiro, 13 de maio de 2008 
Todos os direitos reservados. É proibida a reprodução total ou parcial do trabalho sem autorização da universidade, do autor e do orientador.

\section{Marcus Vinicius Pereira de Souza}

Nascido em Andrelândia-MG, 1972. Graduou-se em Engenharia Elétrica (1999) pela Universidade Federal de Juiz de Fora (UFJF). Mestre em Engenharia Aeronáutica e Mecânica, Área de Produção (2002) pelo Instituto Tecnológico de Aeronáutica (ITA). Suas pesquisas de interesse incluem as áreas de redes neurais artificiais, inteligência artificial, estatística aplicada e pesquisa operacional.

Ficha Catalográfica

Souza, Marcus Vinicius Pereira de

Uma abordagem bayesiana para o cálculo dos custos operacionais eficientes das distribuidoras de energia elétrica / Marcus Vinicius Pereira de Souza ; orientador: Reinaldo Castro Souza. - 2008.

$153 \mathrm{f.} \mathrm{;} 30 \mathrm{~cm}$

Tese (Doutorado em Engenharia Elétrica) - Pontifícia Universidade Católica do Rio de Janeiro, Rio de Janeiro, 2008.

Inclui bibliografia

1. Engenharia elétrica - Teses. 2. Análise envoltória de dados. 3. Fronteiras estocásticas. 4. Rede neural de Kohonen. 5. Regulação econômica. I. Souza, Reinaldo Castro. II. Pontifícia Universidade Católica do Rio de Janeiro. Departamento de Engenharia Elétrica. III. Título. 
Esta Tese é dedicada à memória do meu amado Pai, Sebastião Teixeira de Souza, que certamente está num plano maior intercedendo por mim; e também para minha querida Mãe Elizabeth Meer Pereira de Souza, essencial em todos os momentos da minha vida. 


\section{Agradecimentos}

A Deus, à Nossa Senhora Aparecida, aos Mestres da Grande Fraternidade Branca e a todos os Gloriosos Santos por mais esta conquista.

Consigno meus sinceros agradecimentos e respeito ao professor Dr. Reinaldo Castro Souza pela oportunidade; orientando-me com paciência, dedicação e incentivo em prol desta tese.

Também quero externar o meu apreço sincero ao professor Dr. José Francisco Moreira Pessanha que aconselhou-me e encorajou-me durante toda essa trajetória.

Ao grande amigo e professor Dr. Madiagne Diallo, pelo apoio moral e constante estímulo na concretização desta importante etapa da minha vida.

Quero ainda expressar minha gratidão à Helaine Aparecida da Silva pelos conselhos, incentivos e sua valiosa ajuda na efetivação desta tese.

Muitas outras pessoas contribuíram para o sucesso deste trabalho, em especial: Airam Regina de Aquino Martins, Alexandra Mello Schmidt, Alexandre Bessa dos Santos, Alexandre Zanini, Álvaro Veiga, Ana Paiva, André Luis Marques Marcato, Arildo de Jesus Batista, Armando Zeferino Milioni, Cairo Lúcio Nascimento Jr., Carlos Galeno Ladeira Pereira, Célia Maria de Souza Pereira, Cristiano Augusto C. Fernandes, Eduardo Eliphas Pereira, Ernesto Fagundes Neto, Eurides Moura, Fátima Mendes de Carvalho, Felipe Fortes Carvalho Silva, Fernando César Coelli, Fernando Neves Breseguello, Gisele Teixeira Rocha, Hélio Francisco da Silva, Isnarde Antônio Ernesto, Jacqueline Furtado Vital, José Luiz Bellini Leite, José Marcelino de Jesus, Juliana Garcia Cespedes, Lúcia Helena Lima Miranda e Silva, Lúcia Margareth Pereira, Luiz Tarciso de Andrade, Marcia Brum Guerra Gomes, Márcia Doná, Márcio Vicente Rizzo, Maria Alcina Portes, Maria Aparecida Gonçalves, Mariane Antunes Maia, Marley M. B. Rebuzzi Vellasco, Mischel Carmen Neyra Belderrain, Mônica Barros, Nair de Carvalho Pereira, Neimar Fernandes, Paula Adriana Soares, Pedro Gomes Barbosa, Ricardo Tanscheit, Sebastião Marcos M. P. Vasconcelos, Tatiane Cristina da Silva, Vander Menengoy da Costa, Wilma Galgane.

À PUC-Rio, pelo suporte financeiro do projeto.

Finalmente, mas não menos importante, desejo registrar a minha admiração a todos que me honraram apresentando críticas e sugestões concernentes ao meu trabalho, em particular: Elizabeth Meer Pereira de Souza, Dr. Marcos Azevedo da Silveira, Dra. Mariane Branco Alves e Dr. Tufi Machado Soares. 


\section{Resumo}

Souza, Marcus Vinicius Pereira de; Souza, Reinaldo Castro (Orientador). Uma Abordagem Bayesiana para o Cálculo dos Custos Operacionais Eficientes das Distribuidoras de Energia Elétrica. Rio de Janeiro, 2008. 153p. Tese de Doutorado - Departamento de Engenharia Elétrica, Pontifícia Universidade Católica do Rio de Janeiro

Esta tese apresenta os principais resultados de medidas de eficiência dos custos operacionais de 60 distribuidoras brasileiras de energia elétrica. Baseado no esquema yardstick competition, foi utilizado uma Rede Neural d e Kohonen (KNN) para identificar grupos de empresas similares. Os resultados obtidos pela KNN não são determinísticos, visto que os pesos sinápticos da rede são inicializados aleatoriamente. Então, é realizada uma simulação de Monte Carlo para encontrar os clusters mais frequentes. As medidas foram obtidas por modelos DEA (input oriented, com e sem restrições aos pesos) e modelos Bayesianos e frequencistas de fronteira estocástica (utilizando as funções Cobb-Douglas e Translog). Em todos os modelos, DEA e SFA, a única variável input refere-se ao custo operacional (OPEX). Os índices de eficiência destes modelos representam a potencial redução destes custos de acordo com cada concessionária avaliada. Os outputs são os cost drivers da variável OPEX: número de unidades consumidoras (uma proxy da quantidade de serviço), montante de energia distribuída (uma proxy do produto total) e a extensão da rede de distribuição (uma proxy da dispersão dos consumidores na área de concessão). Finalmente, vale registrar que estas técnicas podem mitigar a assimetria de informação e aprimorar a habilidade do agente regulador em comparar os desempenhos das distribuidoras em ambientes de regulação incentivada.

\section{Palavras-chave}

Análise Envoltória de Dados; Fronteiras Estocásticas, Rede Neural de Kohonen, Regulação Econômica. 


\section{Abstract}

Souza, Marcus Vinicius Pereira de; Souza, Reinaldo Castro (Advisor). A Bayesian Approach to Estimate the Efficient Operational Costs of Electrical Energy Utilities. Rio de Janeiro, 2008. 153p. Doctorate Thesis - Departamento de Engenharia Elétrica, Pontifícia Universidade Católica do Rio de Janeiro

This thesis presents the main results of the cost efficiency scores of 60 Brazilian electricity distribution utilities. Based on yardstick competition scheme, it was applied a Kohonen Neural Networks (KNN) to identify and to group the similar utilities. The KNN results are not deterministic, since the estimated weights are randomly initialized. Thus, a Monte Carlo simulation was used in order to find the most frequent clusters. Therefore was examined the use of the DEA methodology (input oriented, with and without weight constraints) and Bayesian and non-Bayesian Stochastic Frontier Analysis (centered on a CobbDouglas and Translog cost functions) to evaluate the cost efficiency scores of electricity distribution utilities. In both models the only input variable is operational cost (OPEX). The efficiency measures from these models reflect the potential of the reduction of operational costs of each utility. The outputs are the cost-drivers of the OPEX: the number of customers (a proxy for the amount of service), the total electric power supplied (a proxy for the amount of product delivered) and the distribution network size (a proxy of the customers scattering in the operating territory of each distribution utility). Finally, it is important to mention that these techniques can reduce the information assimetry to improve the regulator's skill to compare the performance of the utilities in incentive regulation environments.

\section{Keywords}

Data Envelopment Analysis; Stochastic Frontier Analysis, Kohonen Neural Networks, Economic Regulation. 


\section{Sumário}

$\begin{array}{ll}\text { 1. Introdução } & 18\end{array}$

1.1. Considerações gerais 18

1.2. Revisão bibliográfica $\quad 23$

1.3. Objetivos 24

1.4. Estrutura da tese 26

2. Redes Neurais Auto-Organizáveis 29

2.1. Introdução $\quad 29$

2.2. Componentes de uma tarefa de clustering $\quad 29$

2.3. Aprendizado por competição 31

2.4. Rede Neural de Kohonen (RNK) 32

2.5. Exemplo ilustrativo da Rede Neural de Kohonen 34

2.6. Rede Neural de Kohonen via simulação de Monte Carlo (MCRNK) 38

3. Análise Envoltória de Dados $\quad 40$

3.1. Introdução 40

3.2. Conjunto de Possibilidades de Produção 42

3.3. Conceito de eficiência 44

3.4. Benchmarks $\quad 46$

$\begin{array}{ll}\text { 3.5. Retornos de escala } & 47\end{array}$

3.6. Modelos DEA $\quad 48$

3.6.1. Modelo DEA CCR input-oriented 49

3.6.2. Modelo DEA BCC input-oriented 53

3.6.3. Outros modelos DEA 56

3.6.4. Seleção de variáveis inputs e outputs $\quad 57$

3.6.5. Técnicas baseadas em Restrições aos Pesos 58

3.7. Adjusted contingent restrictions on weights 60

4. Conceitos Básicos de Estatística Bayesiana e Simulação Estocástica 62

4.1. Elementos de inferência Bayesiana 62

4.2. Distribuição a priori $\quad 63$

4.3. Distribuição amostral (Função de Verossimilhança) 64

4.4. Distribução a posteriori 65

4.5. Exemplo 65

4.6. Obtenção de resumos de interesse através de simulação 68

4.6.1. Integração via Monte Carlo 69

4.7. Monte Carlo via Cadeias de Markov (MCMC) 72

4.7.1. Algoritmo de Metropolis-Hastings $\quad 74$

4.7.2. Amostrador de Gibbs $\quad 77$

4.7.3. Monitoração da convergência $\quad 82$

5. Modelos Econométricos $\quad 84$

5.1. Fronteira Estocástica de Produção $\quad 84$

5.2. Fronteira Estocástica de Custos $\quad 89$

5.3. Fronteira Estocástica sob o paradigma Bayesiano 91

5.3.1. Modelos Bayesianos para Fronteira Estocástica 92 
5.3.2. Varying Efficiency Distribution (VED) models

5.4. Inferência Bayesiana usando Amostrador de Gibbs

6. Clustering: Definindo Grupos Estratégicos 98

6.1. Introdução 98

6.2. Análise de dados $\quad 99$

6.3. Resultados obtidos via cluster analysis 99

7. Mensuração da Eficiência Relativa utilizando Modelos DEA 105

7.1. Introdução 105

7.2. Resultados DEA e discussões 105

8. Estimação da Eficiência Técnica usando Fronteiras Estocásticas 110

8.1. Introdução 110

8.2. Resultados dos modelos econométricos com enfoque clássico 110

8.3. Resultados dos modelos econométricos com enfoque Bayesiano 115

9. Análise Multivariada de Dados 123

9.1. Introdução 123

9.2. Matriz de correlação de Pearson e Spearman 123

9.3. Análise Fatorial 127

10. Conclusões e Perspectivas 133

10.1 Conclusões 133

$\begin{array}{ll}10.2 \text { Perspectivas } & 135\end{array}$

$\begin{array}{ll}\text { Referências bibliográficas } & 138\end{array}$

$\begin{array}{ll}\text { Anexo } 1 \text { - Conjunto de Dados } & 149\end{array}$

$\begin{array}{lr}\text { Anexo } 2 \text { - Modelo Bayesiano } & 150\end{array}$ 


\section{Lista de figuras}

Figura 1.1: Processo de revisão tarifária [4] 19

Figura 2.1: Etapas de um processo de clustering $\quad 30$

Figura 2.2: Rede de Kohonen com as unidades de saída organizadas como grade bi-dimensional 33

Figura 2.3: Disposição bi-dimensional das entradas 35

Figura 2.4: Disposição das unidades no espaço das entradas 36

Figura 2.5: Resultado do processo de identificação dos clusters 38

Figura 3.1: Comparação de DEA e regressão [18] 41

Figura 3.2: Eficiências Técnica, Alocativa e Econômica 45

Figura 3.3: Envoltória determinada pelo modelo CCR input-oriented [18] 52

Figura 3.4: Envoltória determinada pelos modelos CCR e BCC (inputoriented) [18] 55

Figura 4.1: Densidades a priori do parâmetro $\theta \quad 66$

Figura 4.2: Função de Verossimilhança para $\theta \quad 67$

Figura 4.3: Densidades a posteriori do parâmetro $\theta \quad 68$

Figura 4.4: Scatterplot dos valores simulados $\quad 71$

Figura 4.5: Valores simulados através do algoritmo Metropolis-Hastings 76

Figura 4.6: Algoritmo Metropolis-Hastings na geração da distribuição de Cauchy

Figura 4.7: Dados simulados para $\lambda$ e $\beta$ utilizando o algoritmo de Gibbs $\quad 80$

Figura 4.8: Resultado das médias amostrais para o parâmetro $\lambda \quad 80$

Figura 4.9: Distribuição marginal estimada de $p(x) \quad 81$

Figura 4.10: Gráficos de autocorrelação amostral dos parâmetros $\lambda$ e $\beta \quad 83$

Figura 5.1: Densidades de probabilidade da combinação de $v_{j}+u_{j} \quad 91$

Figura 6.1: Resultado de um processo de clustering em uma simulação $\quad 100$

Figura 6.2: Histograma dos clusters 101

Figura 6.3: Histograma dos 13 clusters mais freqüentes 103

Figura 7.1: Scatterplot Matrix dos índices de eficiência (Modelos DEA) 108

Figura 8.1: Boxplot dos índices de eficiência (Modelos SFA Clássica) 114

Figura 8.2: Representação gráfica dos índices de eficiência (Modelos Bayesianos) 
Figura 8.3: Estimativa pontual dos coeficientes de regressão para diferentes valores de $r^{*}$

Figura 9.1: Coeficientes de correlação de Pearson e Spearman 125

Figura 9.2: Coeficientes de correlação de Pearson e Spearman 126

Figura 9.3: Coeficientes de correlação de Pearson e Spearman 127

$\begin{array}{ll}\text { Figura 9.4: Gráfico qui-quadrado (Q-Q plot) } & 128\end{array}$

Figura 9.5: Gráfico de dispersão dos loadings (sem rotação ortogonal). 130

Figura 9.6: Gráfico de dispersão dos loadings (com rotação ortogonal). 130

Figura 9.7: Gráfico de dispersão de objetos segundo os factor scores 131 


\section{Lista de tabelas}

Tabela 2.1 - Centro dos clusters com seus respectivos pontos de treinamento

Tabela 3.1 - Representação algébrica de retornos de escala

Tabela 3.2 - Relação entre retornos de escala e elasticidade total da produção

Tabela 6.2 - Clusters obtidos pela Rede de Kohonen em uma simulação 101

$\begin{array}{ll}\text { Tabela } 6.3 \text { - Sequência ordenada dos clusters mais frequentes } & 102\end{array}$

$\begin{array}{ll}\text { Tabela } 6.4 \text { - Agrupamentos finais } & 104\end{array}$

$\begin{array}{ll}\text { Tabela } 7.1 \text { - Variáveis consideradas } & 105\end{array}$

$\begin{array}{ll}\text { Tabela } 7.2 \text { - Índices de eficiência } \theta_{j}^{\text {DEA }} & 107\end{array}$

$\begin{array}{ll}\text { Tabela } 7.3 \text { - Estatística descritiva dos modelos DEA } & 108\end{array}$

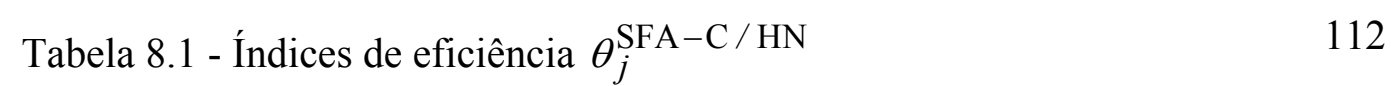

Tabela 8.2 - Índices de eficiência $\theta_{j}^{\mathrm{SFA}-\mathrm{C} / \mathrm{NT}} 113$

$\begin{array}{ll}\text { Tabela } 8.3 \text { - Índices de eficiência } r_{j}^{\mathrm{SFA}-\mathrm{B}} & 117\end{array}$

Tabela 8.4 - Índices de eficiência $r_{j}^{\text {SFA-B }} 118$

Tabela 8.5 - Estimativa dos coeficientes de regressão e parâmetros do modelo de fronteira estocástica (enfoque Bayesiano)

Tabela 8.6 - Índices de eficiência $r_{j}^{\mathrm{SFA}-\mathrm{B} / \mathrm{NT}-\mathrm{TL}} ;\left(r^{*}=0,95\right)$

Tabela 9.1 - Coeficientes de Correlação de Pearson

Tabela 9.2 - Coeficientes de Correlação de Spearman

Tabela 9.3 - Comparação entre os factor loadings não rotacionados e rotacionados

Tabela 9.4 - Ranking das concessionárias 


\section{Símbolos, Acrônimos e Abreviaturas}

d.p. - desvio-padrão;

$e$ - vetor de 1's;

e - Elasticidade Total;

exp - função exponencial;

$\operatorname{Exp}($.$) - distribuição exponencial com parâmetro (.);$

$\mathbf{E}(X)$ - valor esperado da variável aleatória $X$;

$f(x \mid \theta)$ - densidade de $X$, condicional ao parâmetro $\theta$;

$h($.$) - função Translog (TL) ou Cobb-Douglas (CD);$

$i$ - indexador de inputs, $(i=1, \ldots, m)$;

i.i.d - independentes e identicamente distribuídos;

$j$ - indexador de DMU's, $(j=1, \ldots, n)$;

j0 - indexador da DMU que está sendo analisada;

$j t$ - indexador de DMU's eficientes;

$l$ - função de verossimilhança;

ln - logaritmo natural;

$L$ - logaritmo natural da função de verossimilhança;

$m$ - número de inputs;

$\max ($.$) - máximo de (.);$

$\min ($.$) - mínimo de (.);$

$n$ - número de DMU's;

Poi(.) - distribuição de Poisson com parâmetro (.);

$r$ - indexador de outputs, $(r=1, \ldots, s)$;

$\mathbf{R}_{*}^{+}$- Conjunto dos números Reais inteiros positivos;

$\mathbf{R}_{+}^{s}-$ Conjunto dos números Reais inteiros positivos de dimensão $s$;

$\mathbf{R}_{+}^{m}$ - Conjunto dos números Reais inteiros positivos de dimensão $m$;

$s$ - número de outputs (DEA);

T - Conjunto de Possibilidades de Produção;

$u_{r}$ - peso atribuído ao $r$-ésimo output, modelos (DEA) input-oriented; 
$v_{i}$ - peso atribuído ao $i$-ésimo input, modelos (DEA) input-oriented;

$\mathbf{W}_{i}^{k} \quad$ - vetor de pesos sinápticos do neurônio $(i)$ no instante $(k)$;

$X_{j}=$ vetor de variáveis aleatórias (inputs) da DMU $j$ com dimensão 1 x $m$;

$\boldsymbol{X}$ - matriz de inputs observados de dimensão $n \times m$;

$\boldsymbol{x}_{j}=$ vetor de inputs observados da DMU $j$ com dimensão 1 x $m$;

$\boldsymbol{x}=$ vetor de realizações;

$Y_{j}=$ vetor de variáveis aleatórias (outputs) da DMU $j$ com dimensão 1 x $s$;

$\boldsymbol{Y}$ - matriz de outputs observados de dimensão $n \mathrm{xs}$;

$\boldsymbol{y}_{j}=$ vetor de outputs observados da DMU $j$ com dimensão 1 x $s$;

$\boldsymbol{y}=$ vetor de realizações;

$\Gamma(a, b)$ ou Gama(a,b) - distribuição Gama com parâmetros $a$ e $b$;

Unif $(a, b)$ - distribuição Uniforme com parâmetros $a$ e $b$;

$\pi($.$) - distribuição a posteriori;$

[]$^{\mathbf{T}}$ - transposto de [.];

$\boldsymbol{\beta}$ - coeficientes da fronteira estocástica;

$\theta$ - índice de eficiência no modelo DEA CCR e vetor de parâmetros (na abordagem Bayesiana) dependendo do contexto;

$\theta^{*}$ - valor ótimo do índice de eficiência no modelo CCR input-oriented;

$\phi($.$) - função de densidade da Normal-padrão;$

$\Phi($.$) - função de distribuição acumulada da Normal-padrão;$

$\varepsilon$ - infinitésimo positivo ou erro composto dependendo do contexto;

П- produtório;

ANEEL - Agência Nacional de Energia Elétrica;

BCC - Banker, Charnes e Cooper;

CB - Cobb-Douglas;

CCR - Charnes, Cooper e Rhodes;

CED - Common Efficiency Distribution;

DEA - Data Envelopment Analysis;

DMU - Decision Making Unit;

EA - Eficiência Alocativa; 
EE - Eficiência Econômica;

EEF - Equilíbrio Econômico Financeiro;

ET - Eficiência Técnica;

HN - Distribuição Half-Normal;

IRT - Índice de Reajuste Tarifário;

LINDO - Linear Interactive and Discrete Optimizer;

MCMC - Monte Carlo via Cadeias de Markov;

MCRNK - Redes Neurais de Kohonen via simulação de Monte Carlo;

NT - Distribuição Normal-Truncada;

PL - Programação Linear;

PPL - Problema de Programação Linear;

RCE - Retorno Constante de Escala;

RNA - Redes Neurais Artificiais;

RVE - Retorno Variável de Escala;

SEB - Sistema Elétrico Brasileiro;

SFA - Stochastic Frontier Analysis;

TL - Translog;

VED - Varying Efficiency Distribution;

WinBUGS - Bayesian inference Using Gibbs Sampling for Windows. 
"Bem-aventurado o homem que acha Sabedoria, e o homem que adquire Conhecimento."

(Provérbios 3-13) 
"Ninguém ignora tudo, ninguém sabe tudo. Por isso, aprendemos sempre".

(Paulo Freire) 